\title{
Comparison of ${ }^{68} \mathrm{Ga}$-PSMA-I/T PET-CT and Multiparametric MRI for Locoregional Staging of Prostate Cancer Patients: A Pilot Study
}

\author{
Sinan Çelen ${ }^{a} \quad$ Aziz Gültekin ${ }^{b} \quad$ Yusuf Özlülerden $^{a} \quad$ Aslı Meted Ergin Sağtaş $^{c}$

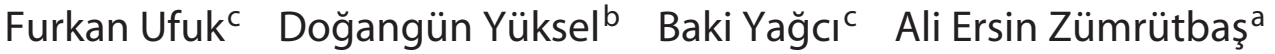

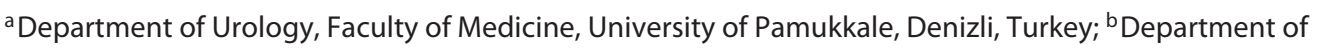 \\ Nuclear Medicine, Faculty of Medicine, University of Pamukkale, Denizli, Turkey; ' Department of Radiology, \\ Faculty of Medicine, University of Pamukkale, Denizli, Turkey; ${ }^{d}$ Department of Anesthesiology, Faculty of \\ Medicine, University of Pamukkale, Denizli, Turkey
}

\section{Keywords}

Prostate cancer $\cdot \mathrm{mp}-\mathrm{MRI} \cdot{ }^{68} \mathrm{Ga}-\mathrm{PSMA}-\mathrm{I} / \mathrm{T}$ PET-CT

\begin{abstract}
Background/Aim: The objective of this work was to assess the value of ${ }^{68} \mathrm{Ga}$-DOTAGA-(3-iodo-y)fk(Sub-KuE) positron emission tomography ( ${ }^{68} \mathrm{Ga}-\mathrm{PSMA}-\mathrm{I} / \mathrm{T}$ PET-CT) and multiparametric magnetic resonance imaging (mp-MRI) for preoperative staging in prostate cancer ( $\mathrm{PCa}$ ) patients who underwent radical prostatectomy (RP) by validating with postoperative histopathology data. Materials and Methods: We prospectively investigated 30 consecutive $P C$ a patients who had both $\mathrm{mp}-\mathrm{MRI}$ and ${ }^{68} \mathrm{Ga}-\mathrm{PSMA}-\mathrm{I} / \mathrm{T}$ PET-CT before laparoscopic RP. The seminal vesicle invasion (SVI), lymph node metastasis (LNM), bladder neck invasion (BNI), and extracapsular extension (ECE) were investigated separately. The diagnostic performances of $\mathrm{mp}-\mathrm{MRI}$ and ${ }^{68} \mathrm{Ga}-\mathrm{PSMA}-\mathrm{I} / \mathrm{T}$ PET-CT were assessed using histopathological results. Results: Both mp-MRI and ${ }^{68} \mathrm{Ga}-\mathrm{PSMA}-\mathrm{I} / \mathrm{T}$ PET-CT were not statistically significant in the evaluation of SVI, BNI, and ECE preoperatively but had statistically significant results in the assessment of LNM. mp-MRI had higher overall sensitivity for ECE, overall
\end{abstract}

karger@karger.com

(c) 2020 S. Karger AG, Basel

www.karger.com/uin

Karger specificity for SVI, ECE, and BNI, and positive predictive value for ECE, SVI, BNI. ${ }^{68} \mathrm{Ga}-\mathrm{PSMA}-\mathrm{I} / \mathrm{T}$ PET-CT had higher overall sensitivity for $\mathrm{BNI}$, and negative predictive value for $\mathrm{BNI}$ and LNM. Conclusion: mp-MRI has superior specificity, sensitivity, and accuracy for assessing ECE and SVI. Both imaging modalities had similar specificity, sensitivity, and accuracy for determining BNI. However, both imaging modalities had low diagnostic accuracy for LNM on histopathology.

(c) 2020 S. Karger AG, Basel

\section{Introduction}

Prostate cancer ( $\mathrm{PCa}$ ) is the most frequent type of malignancy in men and has the second highest mortality rate among male malignant carcinomas. Radical prostatectomy (RP) is one of the commonly used first-line treatment modalities for patients with localized PCa [1]. Current European guidelines recommend MRI for local staging and $\mathrm{CT} /$ bone scan to achieve accurate staging for those with distant metastases before treatment of PCa [2]. However, these conventional imaging modalities have low sensitivity and specificity to identify small lesions. 
Moreover, misdiagnosis of benign lesions may lead to incorrect treatment or overtreatment in non-metastatic PCa patients. Novel imaging modalities, such as ${ }^{68} \mathrm{Ga}-D O T A G A-\left(3\right.$-iodo-y)fk(Sub-KuE) ${ }^{68} \mathrm{Ga}-\mathrm{PSMA}-\mathrm{I} /$ T PET-CT) and multiparametric magnetic resonance imaging (mp-MRI; including diffusion-weighted imaging [DWI] and dynamic contrast-enhanced T1-weighted imaging), have been developed for the treatment planning of PCa patients [2].

MRI plays an essential role in detecting suspicious lesions for $\mathrm{PCa}$, guiding the biopsy procedure, and local staging in biopsy-proven PCa patients. Recent advances in mp-MRI have led to the detection of clinically significant disease and reduced unnecessary biopsies and treatment [3-7]. mp-MRI, including T1- and T2-weighted DWI and T1-weighted dynamic contrast-enhanced sequences, is used to increase the diagnostic accuracy of the MRI for PCa [8-13]. This modality is also widely used for active surveillance and guiding the repeat biopsies in these patients [14].

Prostate-specific membrane antigen (PSMA) is a type II transmembrane glycoprotein that is expressed in normal prostate gland epithelium and overexpressed on the surface of $>80 \%$ of the primary and metastatic PCa $[15$, 16]. ${ }^{68} \mathrm{Ga}$-PSMA 11 binds to the receptor of transmembrane folate hydrolase and can be imaged using positron emission tomography-computed tomography (PET-CT) scan $[17,18]$. Recently, ${ }^{68}$ Ga-PSMA-I/T PET-CT has been used in PCa detection, diagnosis, and staging [19, 20]. However, no study has so far compared the diagnostic performance of mp-MRI and ${ }^{68} \mathrm{Ga}$-PSMA-I/T PET$\mathrm{CT}$. Therefore, we aimed to compare the diagnostic performance of ${ }^{68} \mathrm{Ga}$-PSMA-I/T PET-CT and mp-MRI in $\mathrm{PCa}$, using histopathological findings as the gold standard.

\section{Materials and Methods}

\section{Study Population}

The study protocol was approved by Pamukkale University School of Medicine Human Research Ethics Committee (approval No. 60116787-020/56122) and the study was conducted ethically in accordance with the World Medical Association Declaration of Helsinki. Patients who agreed to participate in the study and planned to undergo laparoscopic RP were included and patients underwent both mp-MRI and ${ }^{68} \mathrm{Ga}-\mathrm{PSMA}-\mathrm{I} / \mathrm{T}$ PET-CT preoperatively. After imaging, patients underwent laparoscopic RP (LRP) within 3 days by a single urologist with 5 years of experience. Individuals with a history of treated PCa and a concurrent diagnosis of any other primary malignancy were excluded from the study. Additionally, patients who had more than the 6-week time interval between imaging to LRP and PSMA uptake related to PCa within
Table 1. Clinical and pathological data of the patients

\begin{tabular}{lc}
\hline Age, years $^{1}$ & $65.07 \pm 8.01(46-82)$ \\
Preoperative PSA $(n=30), \mathrm{ng} / \mathrm{mL}$ & $9.49 \pm 6.97(1.3-27)$ \\
Postoperative Gleason score $(n=30)$ & \\
$\quad 3+3$ & $7(23.3)$ \\
$3+4$ & $7(23.3)$ \\
$4+3$ & $5(16.7)$ \\
$4+4$ & $1(3.3)$ \\
$3+5$ & $1(3.3)$ \\
$4+5$ & $2(6.7)$ \\
$5+3$ & $1(3.3)$ \\
$5+4$ & $3(10)$ \\
$5+5$ & $3(10)$ \\
D’Amico risk stratification $^{\prime}$ & \\
Low & $2(6.7)$ \\
Intermediate & $3(10)$ \\
High & $25(83.3)$ \\
LNM & \\
$\quad$ Negative & $21(95.5)$ \\
Positive & $1(4.5)$ \\
SVI & $6(20)$ \\
ECE & $17(56.7)$ \\
BNI & $4(13.3)$ \\
mp-MRI PI-RADS score & \\
2 & $1(3.3)$ \\
4 & $11(36.7)$ \\
5 & $18(60)$ \\
${ }^{1}$ Ga-PSMA-I/T PET-CT SUV max $^{2} \mathrm{~T}^{1}$ & $11.25 \pm 11.03(2.2-52.66)$ \\
\end{tabular}

Data are presented as the mean \pm SD (range) or $n(\%)$. BNI, bladder neck invasion; PSA, prostate-specific antigen; PI-RADS, Prostate Imaging - Reporting and Data System; ECE, extracapsular extension; mp-MRI, multiparametric magnetic resonance imaging; SVI, seminal vesicle invasion; LNM, lymph node metastasis; $\mathrm{SUV}_{\max }-\mathrm{T}$, maximum standard uptake value of tumor.

${ }^{1} n=30 .{ }^{2} n=22$.

the distant metastases were excluded. In all patients the TNM (tumor, node, and metastasis) system was used for PCa staging [21]. Patients with confirmed PCa were stratified by the D'Amico risk stratification [22].

mp-MRI Examination and Analysis

mp-MRI was performed using a 1.5 -T superconducting magnet (Ingenia, Philips Medical Systems, The Netherlands) and a 32-channel torso coil. Transverse, coronal, and sagittal T2-weighted turbo spin-echo images were acquired, respectively (3-mm slice thickness without gap, 2,290 ms time-to-repetition, $100 \mathrm{~ms}$ timeto-echo, number of signals acquired 2). Axial DWI were obtained using spin-echo echo-planar imaging sequences and two b-values (3-mm slice thickness without gap, 4,000 ms time-to-repetition, 90 $\mathrm{ms}$ time-to-echo, number of signals acquired 12 , and b-values were 0 and $1,800 \mathrm{~s} / \mathrm{mm}^{2}$ ). Fat-suppressed T1-weighted isovolumetric images after intravenous gadolinium-based contrast medium (0.1 $\mathrm{mmol} / \mathrm{kg}$, gadoterate meglumine, Dotarem, Guerbet, France) injection were obtained for dynamic contrast-enhanced images (6$\mathrm{mm}$ slice thickness, intersection gap $3 \mathrm{~mm}, 3.9 \mathrm{~ms}$ time-to-repeti- 
Table 2. Relationship between histopathological features and imaging findings

\begin{tabular}{|c|c|c|c|c|c|c|c|c|}
\hline & \multicolumn{2}{|l|}{ SVI } & \multicolumn{2}{|l|}{ ECE } & \multicolumn{2}{|l|}{ BNI } & \multicolumn{2}{|l|}{ LNM } \\
\hline \multicolumn{9}{|c|}{ D'Amico risk stratification } \\
\hline Low risk & - & 2 & - & 2 & - & 2 & - & - \\
\hline Intermediate risk & - & 3 & - & 3 & - & 3 & - & 1 \\
\hline High risk & 6 & 19 & 17 & 8 & 4 & 21 & 1 & 20 \\
\hline $3+3$ & - & 7 & 1 & 6 & - & 7 & - & 3 \\
\hline $3+4$ & - & 7 & 4 & 3 & - & 7 & - & 4 \\
\hline $4+3$ & 2 & 3 & 3 & 2 & 1 & 4 & - & 5 \\
\hline $4+4$ & 1 & - & 1 & - & - & 1 & - & 1 \\
\hline $3+5$ & - & 1 & 1 & - & - & 1 & - & 1 \\
\hline $4+5$ & - & 2 & 1 & 1 & - & 2 & - & 2 \\
\hline PI-RADS 2 & - & 1 & - & 1 & - & 1 & - & - \\
\hline PI-RADS 3 & - & - & - & - & - & - & - & - \\
\hline PI-RADS 4 & - & 11 & 4 & 7 & - & 11 & - & 5 \\
\hline PI-RADS 5 & 6 & 12 & 13 & 5 & 4 & 14 & 1 & 16 \\
\hline \multicolumn{9}{|l|}{$\mathrm{SUV}_{\max }-\mathrm{T}$} \\
\hline Mean \pm SD & $17.88 \pm 8.07$ & $9.59 \pm 11.17$ & $11.57 \pm 8.76$ & $10.83 \pm 13.82$ & $24.35 \pm 5.77$ & $9.23 \pm 10.26$ & 31.89 & $13.26 \pm 11.20$ \\
\hline$p$ value & 0.01 & & 0.213 & & 0.001 & & 0.120 & \\
\hline \multicolumn{9}{|c|}{ Preoperative PSA, ng/mL } \\
\hline Mean \pm SD & $14.53 \pm 10.44$ & $8.22 \pm 5.41$ & $10.56 \pm 7.07$ & $8.08 \pm 6.86$ & $12.88 \pm 10.94$ & $8.96 \pm 6.32$ & 27 & $9.76 \pm 6.96$ \\
\hline$p$ value & 0.045 & & & 0.345 & & 0.659 & & 0.098 \\
\hline
\end{tabular}

A $p$ value $\leq 0.05$ is considered statistically significant. SVI, seminal vesicle invasion; ECE, extracapsular extension; BNI, bladder neck invasion; LNM, lymph node metastasis; PI-RADS, Prostate Imaging - Reporting and Data System; PSA, prostate-specific antigen; mp-MRI, multiparametric magnetic resonance imaging; $\mathrm{SUV}_{\max }-\mathrm{T}$, maximum standard uptake value of tumor.

tion, 1.83 ms time-to-echo, number of signals acquired 1, 7-s dynamic scan time). Apparent diffusion coefficient maps were generated from DWI images using a dedicated software (Extended Workspace, Philips Healthcare, The Netherlands). MR images were evaluated by a urogenital radiologist with 22 years of experience (Observer 1) who was unaware of the clinical features, pathology, and PET-CT results of the patients. The Prostate Imaging Reporting and Data System (PI-RADS) version 2 was used to calculate mp-MRI scores [23].

\section{${ }^{68} \mathrm{Ga}-P S M A-I / T$ PET-CT Examination and Analysis}

The ${ }^{68} \mathrm{Ga}-\mathrm{PSMA}-\mathrm{I} / \mathrm{T}$ was carried out using a Scintomics synthesis unit (Lindach, Fürstenfeldbruck, Germany) by a qualified radiochemist under the recommended laboratory conditions. Quality control of the ${ }^{68} \mathrm{Ga}-\mathrm{PSMA}-\mathrm{I} / \mathrm{T}$ radionuclide was performed by high-performance liquid chromatography and thin layer chromatography, validated according to previously described methods $[19,20]$, after a single ${ }^{68} \mathrm{Ga}-\mathrm{PSMA}-\mathrm{I} / \mathrm{T}$ injection (mean $185 \mathrm{MBq}$; range $125-317 \mathrm{MBq}$ ) followed by a 60-min uptake period. Then, image acquisitions were carried out while the patient was lying in the supine position. PET-CT images were obtained using a PET-CT unit (Gemini TF TOF PET-CT; Philips, Cleveland, $\mathrm{OH}, \mathrm{USA}$ ). No intravenous contrast was used during emis- sion scans, and image acquisition was from the skull vertex to knee. The transmission images were obtained using a low-dose CT protocol (50-120 mA, 90-140 kV, 5-mm slice thickness). For the PET images, attenuation correction was performed using CT features and the ordered subsets-expectation maximization algorithm (33 subsets, three iterations). The iterative method was used to reconstruct the PET images.

\section{Evaluation of ${ }^{68} \mathrm{Ga}-\mathrm{PSMA}-\mathrm{I} / \mathrm{T}$ PET-CT}

Two observers (Observer 2 and Observer 3) with 12 years of experience in PET-CT imaging, and who were unaware to the clinical features, pathology, and PET-CT results of the patients, evaluated the images, reaching a consensus. Image analysis was performed qualitatively and semiquantitatively. For prostate gland lesions to be considered positive when focal uptake was performed on the background prostatic uptake, values of maximum standardized uptake $\left(\mathrm{SUV}_{\max }\right)$ normalized to body weight were used to assess the ${ }^{68} \mathrm{Ga}-\mathrm{PSMA}-\mathrm{I} / \mathrm{T}$ uptake in the primary tumor focus. The $\mathrm{SUV}_{\text {max }}$ value in the prostate was noted at the site of the most intense uptake in the prostate gland. Seminal vesicle invasion (SVI), extracapsular extension (ECE), lymph node metastasis (LNM), and bladder neck invasion (BNI) were evaluated visually in each patient. 
Table 3. Relationship between histopathological features and imaging findings

\begin{tabular}{llc}
\hline $\begin{array}{l}\text { Postoperative } \\
\text { Gleason score }\end{array}$ & $\begin{array}{l}\text { mp-MRI PI-RADS, } \\
\end{array}$ & $\begin{array}{c}{ }^{68} \text { Ga-PSMA- } \\
\text { I/T PET-CT } \\
\text { SUV } \text { max }^{-T}\end{array}$ \\
\hline $3+3(n=7)$ & PI-RADS $2=1$ & $3.84 \pm 2.48$ \\
& PI-RADS $4=5$ & \\
$3+4(n=7)$ & PI-RADS $5=1$ & $10.81 \pm 18.47$ \\
$4+3(n=5)$ & PI-RADS $4=3$ & $16.02 \pm 4.98$ \\
$4+4(n=1)$ & PI-RADS $5=4$ & 13.76 \\
$3+5(n=1)$ & PI-RADS $5=5$ & 2.67 \\
$4+5(n=2)$ & PI-RADS $5=1$ & $11.39 \pm 0.23$ \\
$5+3(n=1)$ & PI-RADS $5=1$ & 4.75 \\
$5+4(n=3)$ & PI-RADS $4=1$ & $12.87 \pm 5.85$ \\
$5+5(n=3)$ & PI-RADS $5=1$ & \\
& PI-RADS $4=1$ & $24.04 \pm 8.78$ \\
\hline
\end{tabular}

mp-MRI, multiparametric magnetic resonance imaging; PIRADS, Prostate Imaging - Reporting and Data System; $S_{\text {UV }}-\mathrm{T}$, maximum standard uptake value of tumor.

\section{Surgery}

All LRP operations were performed by a urologist who had 5 years of experience in LRP. An extraperitoneal antegrade laparoscopic approach was performed as previously described with some modifications [24].

After abdominal access and entering of the Retzius space, the endopelvic fascia was opened and the dorsal vein complex reached. After ligation of the dorsal vein complex, incision of the bladder neck was performed and on reaching the vas deferens dissection was completed. Controlling the prostatic pedicles, neurovascular bundle dissection was performed towards the urethra. Afterwards, the urethra was cut and by removing the prostate from the area urethrovesical anastomosis was performed. Pelvic lymphadenectomy was also performed in those who had an intermediate or high risk of $\mathrm{PCa}$.

\section{Histopathological Examination}

The pathological evaluation of each case was reviewed by a uropathologist with 10 years of experience in uropathology. The evaluation of prostate histopathology was made according to the 2014 International Society of Urological Pathology (ISUP) [25]. ECE, SVI, BNI, LNM, and the number of positive lymph nodes were recorded.

\section{Statistical Analysis}

The diagnostic performance of mp-MRI and ${ }^{68} \mathrm{Ga}-\mathrm{PSMA}-\mathrm{I} / \mathrm{T}$ PET-CT in detecting ECE, SVI, LNM, and BNI were verified using the histopathological examination. All demographic data analyses were performed using an independent $\chi^{2}$ with significance at the 0.05 level. The one-sample $t$ test and the $\chi^{2}$ test were used to analyze the correlation between mp-MRI, ${ }^{68} \mathrm{Ga}$-PSMA-I/T PET-CT, and histopathological findings. The McNemar $\chi^{2}$ test was used to compare the sensitivity and specificity of diagnostic examinations.

Comparison of ${ }^{68} \mathrm{Ga}-\mathrm{PSMA}-\mathrm{I} / \mathrm{T}$ PET-CT

and $\mathrm{mp}-\mathrm{MRI}$

\section{Results}

Clinical data of the patients are presented in Table 1. Histopathologically, all cases were acinar adenocarcinomas. The mean age of the patients was $65.07 \pm 8.01$ years (range 46-82). The mean serum prostate-specific antigen (PSA) level was $9.49 \pm 6.97 \mathrm{ng} / \mathrm{mL}$ (range 1.3-27). Among the 30 patients, SVI, BNI, and ECE were present in 6 (20\%), 4 (13.3\%), and $17(56.7 \%)$ cases, respectively. Lymph node dissection was performed in 22 patients, and 1 patient had lymph node metastasis.

Most of the patients (96.7\%) had PI-RADS 4 and 5 lesions in mp-MRI. All of the patients with SVI, ECE, LNM, and who were BNI positive were in the high-risk group. PI-RADS 5 lesions were reported in all patients who had a positive SVI and BNI status and also PI-RADS 4 and 5 lesions were reported in all patients who had a positive ECE status. Moreover, preoperative PSA levels and $\mathrm{SUV}_{\max }-\mathrm{T}$ values were significantly higher in patients with SVI, and SUV $\mathrm{max}-\mathrm{T}$ was higher in patients with BNI $(p<0.05$; Table 2).

Table 3 shows the association between imaging variables and Gleason scores. Both mp-MRI and ${ }^{68} \mathrm{Ga}-\mathrm{PS}-$ MA-I/T PET-CT had no statistically significant results for the assessment of SVI, BNI, and ECE-positive or negative tumors according to prostate pathology. However, both ${ }^{68} \mathrm{Ga}$-PSMA-I/T PET-CT LNM and mp-MRI predicted LNM significantly. Although statistically not significant, mp-MRI had higher overall sensitivity for ECE, overall specificity for SVI, ECE, and BNI, and higher positive predictive value (PPV) for ECE, SVI, and BNI. Furthermore, ${ }^{68} \mathrm{Ga}$-PSMA-I/T PET-CT had higher overall sensitivity for BNI and negative predictive value (NPV) for BNI and LNM, as shown in Table 4 and Figures 1 and 2.

\section{Discussion}

An accurate test must provide valuable information about the initial evaluation, diagnosis, and staging of patients with suspected PCa. Specificity and sensitivity are much more critical with cancer imaging modalities because false-positive results create anxiety and lead to unnecessary and invasive follow-up tests such as biopsies. The most valuable imaging technique for the diagnosis and staging is still controversial for patients with $\mathrm{PCa}$.

Despite a small number of patients, to the best of our knowledge this is the first study to compare both ${ }^{68} \mathrm{Ga}$ PSMA-I/T PET-CT and mp-MRI to histological findings 
Fig. 1. Prostate mp-MRI of a 72-year-old patient with a PSA value of $36.16 \mathrm{ng} / \mathrm{mL}$ and Gleason score of $4+3 \mathrm{PCa}$. The sagittal (a), coronal (b), and axial (c) T2-weighted, high $b$-value $\left(1,800 \mathrm{~s} / \mathrm{mm}^{2}\right)$ diffusionweighted (d), apparent diffusion coefficient map (e), and dynamic contrast-enhanced (f) MRI images reveal a PI-RADS score of 5 lesions in the right peripheral zone, with extraprostatic extension and right SVI.
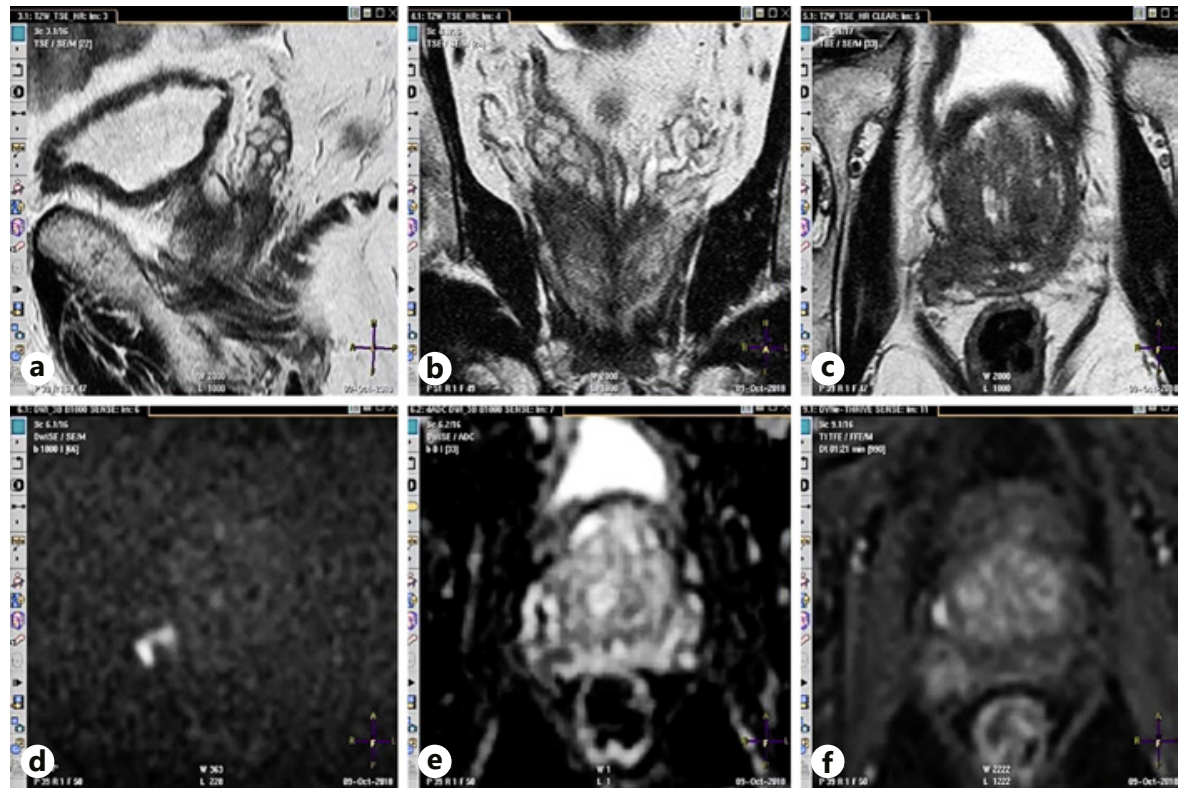

Table 4. Diagnostic accuracy of ${ }^{68} \mathrm{Ga}-\mathrm{PSMA}-\mathrm{I} / \mathrm{T}$ PET-CT and mp-MRI

\begin{tabular}{|c|c|c|c|c|c|c|}
\hline & $\begin{array}{l}\text { Sensitivity, } \\
\%\end{array}$ & $\begin{array}{l}\text { Specificity, } \\
\%\end{array}$ & $\begin{array}{l}\text { PPV, } \\
\%\end{array}$ & $\begin{array}{l}\mathrm{NPV} \\
\%\end{array}$ & $\begin{array}{l}\text { Accuracy, } \\
\%\end{array}$ & $p$ \\
\hline \multicolumn{7}{|l|}{ SVI } \\
\hline mp-MRI & 83.3 & 87.5 & 62.5 & 95.45 & 86.6 & 0.625 \\
\hline${ }^{68} \mathrm{Ga}-\mathrm{PSMA}-\mathrm{I} / \mathrm{T}$ PET-CT & 83.33 & 79.17 & 50 & 95 & 80 & 0.219 \\
\hline \multicolumn{7}{|l|}{ ECE } \\
\hline mp-MRI & 76.47 & 61.54 & 72.22 & 66.67 & 70 & 0.383 \\
\hline${ }^{68} \mathrm{Ga}-\mathrm{PSMA}-\mathrm{I} / \mathrm{T}$ PET-CT & 53.94 & 53.85 & 60 & 46.67 & 53.33 & 0.791 \\
\hline \multicolumn{7}{|l|}{ BNI } \\
\hline mp-MRI & 50 & 96.15 & 66.67 & 92.59 & 90 & 1 \\
\hline${ }^{68} \mathrm{Ga}-\mathrm{PSMA}-\mathrm{I} / \mathrm{T}$ PET-CT & 100 & 88.46 & 57.14 & 100 & 90 & 0.250 \\
\hline \multicolumn{7}{|l|}{ LNM } \\
\hline $\mathrm{mp}-\mathrm{MRI}$ & 100 & 42.86 & 7.69 & 100 & 45.45 & 0.000 \\
\hline${ }^{68} \mathrm{Ga}-\mathrm{PSMA}-\mathrm{I} / \mathrm{T}$ PET-CT & 100 & 47.62 & 8.33 & 100 & 50 & 0.000 \\
\hline
\end{tabular}

mp-MRI, multiparametric magnetic resonance imaging; SVI, seminal vesicle invasion; ECE, extracapsular extension; BNI, bladder neck invasion; LNM, lymph node metastases; PPV, positive predictive value; NPV, negative predictive value.

from the RP specimen. Preoperative accurate assessment of lymph node involvement, SVI, ECE, and BNI status is critical in intermediate- to high-risk PCa patients. Unfortunately, the most commonly used conventional imaging has limited specificity and sensitivity for preoperative assessment, so the clinical status of the patients is largely underestimated with these traditional techniques [26, 27]. We evaluated the potential of ${ }^{68} \mathrm{Ga}$-PSMA-I/T PETCT for imaging primary PCa by intra-individual comparison to mp-MRI. Our results showed that both the
${ }^{68} \mathrm{Ga}$-PSMA-I/T PET-CT and mp-MRI had a significantly high sensitivity to detect LNM. However, ${ }^{68} \mathrm{Ga}$-PSMAI/T PET-CT and mp-MRI showed no statistically significant results for detecting SVI, BNI, and ECE. mp-MRI had higher overall sensitivity for ECE, overall specificity for SVI, ECE, and BNI, and PPV for ECE, SVI, and BNI. ${ }^{68} \mathrm{Ga}-\mathrm{PSMA}-\mathrm{I} / \mathrm{T}$ PET-CT had higher overall sensitivity for BNI and NPV for BNI and LNM. A comparison of imaging modalities to histopathologic results was the fundamental strength of this study. 
Fig. 2. ${ }^{68} \mathrm{Ga}-\mathrm{PSMA}-\mathrm{I} / \mathrm{T}$ PET-CT of a 73-year-old patient with PCa (Gleason score $5+4=9$, grade group 5, ISUP 2014 and preoperative PSA $20 \mathrm{ng} / \mathrm{mL}$ ). a Maximum intensity projection image of pretreatment. b, c Transaxial fused PET-CT and CT images with a gross tumoral lesion (green arrows). d, e Transaxial fused PETCT and CT images establishing right SVI

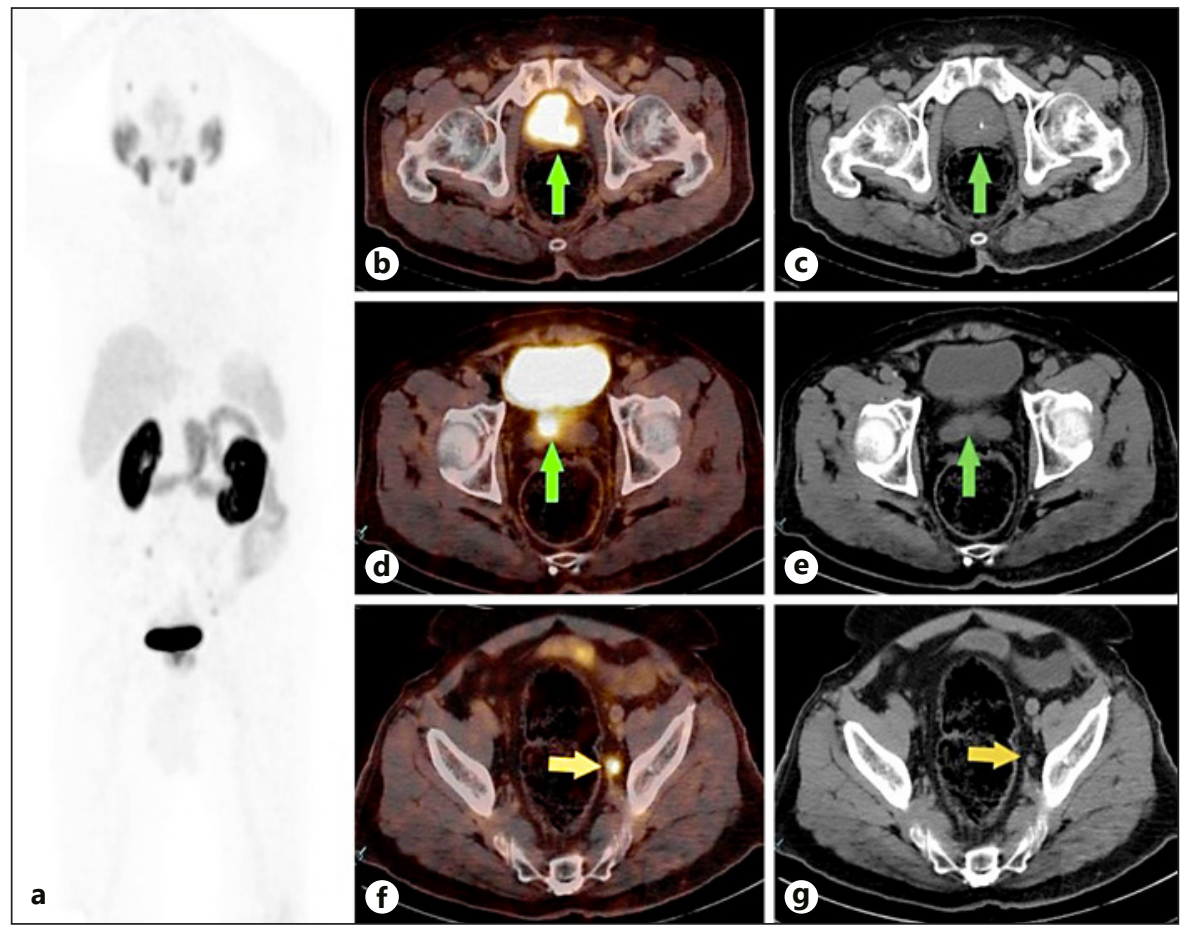

Assessment of SVI and ECE

Evaluation of SVI and ECE in the case of PCa is critical to determine the correct therapeutic approach because SVI or ECE invasion are crucial prognostic factors for recurrence after RP. Patients with an advanced local disease with ECE and SVI generally have a worse prognosis because the risk of a positive surgical margin and the incidence of LNM is increased [28]. Berger et al. [29] reported that specificity of both PSMA PET-CT and mp-MRI were similar for diagnosing SVI (92.7 vs. 95.0\%, $p=0.39$ ). Still, mp-MRI showed superior sensitivity in detecting SVI (75.0\%) compared to PSMA PET-CT (11.1\%). Yilmaz et al. [30] showed that the SVI assessment of mp-MRI was superior to ${ }^{68} \mathrm{Ga}$-PSMA-11 PET-CT, although PET-CT and MRI were associated with clinically and statistically significant results. In our study, ${ }^{68} \mathrm{Ga}$-PSMA-I/T PET-CT and mp-MRI were similar in terms of sensitivity, but specificity was superior with mp-MRI over ${ }^{68} \mathrm{Ga}$-PSMAI/T PET-CT. However, the results were not statistically significant, probably due to the number of included patients. For further confirmation, studies with a higher number of patients are needed.

The presence of ECE is a poor prognostic factor and known to increase the risk of PCa-related mortality [31]. Furthermore, nerve-sparing surgery should be performed to avoid positive surgical margins at the high-risk area of
ECE [2]. Reported sensitivity and specificity of mp-MRI in the detection of ECE was not found to be sufficient (57\% in a meta-analysis) [27]. However, the diagnostic performance of ${ }^{68} \mathrm{Ga}$-PSMA PET-CT in the detection of EPE remains unclear. Prompt diagnosis of ECE is essential for the decision to perform nerve-sparing surgery and is a further benefit for preoperative use of ${ }^{68} \mathrm{Ga}$-PSMA PET-CT. Yilmaz et al. [30] reported that the determination of EPE status was significantly possible using mp-MRI, which had a considerable range in the accuracy (87.5\%), specificity (85.7\%), and sensitivity (90\%) that was verified with the final pathology findings for RP. In the current study, we also found that the sensitivity, specificity, PPV, and NPV of mp-MRI were higher for the detection of ECE. However, these results were not statistically significant.

\section{Assessment of BNI}

Our study showed that both mp-MRI and ${ }^{68} \mathrm{Ga}$-PSMAI/T PET-CT had a similar and relatively inadequate ability to determine BNI accurately. Yilmaz et al. [30] found that mp-MRI and ${ }^{68} \mathrm{Ga}$-PSMA PET-CT showed similar sensitivity, specificity, PPV, NPV, and accuracy in the diagnosis of BNI $(33.3,100,100,81.8$, and $83.3 \%$, respectively). ${ }^{68} \mathrm{Ga}$-PSMA PET-CT has the disadvantage of bladder activity, which can be a problem for the assessment of BNI [26]. Since there are limited data in the pub- 
lished literature on this topic, it is currently not possible to compare our data with others until further studies are published, preferably with a higher number of patients.

\section{Assessment of LNM}

LNM on routine histology is a robust negative predictor of survival [32]. Therefore, evaluation of lymph nodes should be performed optimally before treatment decisions are made. Petersen et al. [33] reported that both MRI and $\mathrm{CT}$ as well as DW-MRI had a lower sensitivity compared with ${ }^{68} \mathrm{Ga}$-PSMA PET-CT. MRI/CT (8 and 100\%) and DW-MRI (36 and 83\%) had lower sensitivity and specificity for LNM detection compared to ${ }^{68} \mathrm{Ga}$-PSMA PET-CT (39 and 100\%, retrospectively). In general, the sensitivity of ${ }^{68} \mathrm{Ga}$-PSMA PET-CT was about $66 \%$ in most trials at the patient level. Moreover, ${ }^{68} \mathrm{Ga}$-PSMA PET-CT has a high specificity (98.9\%) and high accuracy (88.5\%) [34]. In the current study, ${ }^{68} \mathrm{Ga}-\mathrm{PSMA}-\mathrm{I} / \mathrm{T}$ PET-CT and mpMRI were similar and able to predict LNM in terms of sensitivity, specificity, PPV, NPV, and accuracy. However, our positive lymph node dissection group was limited.

Our study is not without limitations. Firstly, due to its retrospective nature, patients represented a heterogeneous group, and the study included a limited number of patients. The small number of patients with positive lymph nodes was another limiting factor. The results need to be validated in a prospective study comprising a larger dataset.

In this study, we demonstrated that both mp-MRI ${ }^{68} \mathrm{Ga}$-PSMA PET-CT had significant results for detecting LNM in patients with PCa. Although the results for detecting ECE, SVI, and BNI were not statistically significant due to the limited number of patients, mp-MRI had superior specificity, sensitivity, NPV, PPV, and accuracy for assessing ECE and SVI in patients with PCa when compared to ${ }^{68} \mathrm{Ga}$-PSMA PET-CT overall. For the detection of BNI, however, ${ }^{68} \mathrm{Ga}$-PSMA PET-CT had higher sensitivity and NPV, and mp-MRI had higher specificity and PPV. We suggest that the use of mp-MRI and ${ }^{68} \mathrm{Ga}$ PSMA PET-CT as new and advanced imaging modalities is a promising field in the management of patients with $\mathrm{PCa}$, and further studies with higher numbers are urgently required to provide a more accurate conclusion and enable these modalities to be used more widely.

\section{Statement of Ethics}

All patients included in this study gave written informed consent. This study complies with the guidelines for human studies and animal welfare regulations. This study protocol was approved by Pamukkale University School of Medicine Human Research Ethics Committee (approval No. 60116787-020/56122) and the study was conducted ethically in accordance with the World Medical Association Declaration of Helsinki.

\section{Conflict of Interest Statement}

The authors have no conflicts of interest to declare.

\section{Funding Sources}

No funding was acquired for this work.

\section{Author Contributions}

Sinan Çelen: protocol development, data collection, data analysis, and manuscript writing. Aziz Gültekin, Yusuf Özlülerden, Aslı Mete, Ergin Sağtaş, Baki Yağcı: data collection. Furkan Ufuk, Ali Ersin Zümrütbaş, Doğangün Yüksel: protocol development and manuscript editing.

\section{References}

1 Cary KC, Punnen S, Odisho AY, Litwin MS, Saigal CS, Cooperberg MR; NIDDK Urologic Diseases in America Project. Nationally representative trends and geographic variation in treatment of localized prostate cancer: the Urologic Diseases in America project. Prostate Cancer Prostatic Dis. 2015 Jun;18(2): $149-54$.

2 Mottet N, Bellmunt J, Bolla M, Briers E, Cumberbatch MG, De Santis M, et al. EAU-ESTRO-SIOG Guidelines on Prostate Cancer. Part 1: Screening, Diagnosis, and Local Treatment with Curative Intent. Eur Urol. 2017 Apr;71(4):618-29.
3 Manfredi M, DE Luca S, Fiori C. Multiparametric prostate MRI for prostate cancer diagnosis: is this the beginning of a new era? Minerva Urol Nefrol. 2017 Dec;69(6):628-9.

4 Monni F, Fontanella P, Grasso A, Wiklund P, Ou YC, Randazzo M, et al. Magnetic resonance imaging in prostate cancer detection and management: a systematic review. Minerva Urol Nefrol. 2017 Dec;69(6):567-78.

5 Siddiqui MM, Rais-Bahrami S, Truong $\mathrm{H}$, Stamatakis L, Vourganti S, Nix J, et al. Magnetic resonance imaging/ultrasound-fusion biopsy significantly upgrades prostate cancer versus systematic 12-core transrectal ultrasound biopsy. Eur Urol. 2013 Nov;64(5):713-9.
6 Salami SS, Ben-Levi E, Yaskiv O, Ryniker L, Turkbey B, Kavoussi LR, et al. In patients with a previous negative prostate biopsy and a suspicious lesion on magnetic resonance imaging, is a 12-core biopsy still necessary in addition to a targeted biopsy? BJU Int. $2015 \mathrm{Apr}$; 115(4):562-70.

7 Vourganti S, Rastinehad A, Yerram N, Nix J, Volkin D, Hoang A, et al. Multiparametric magnetic resonance imaging and ultrasound fusion biopsy detect prostate cancer in patients with prior negative transrectal ultrasound biopsies. J Urol. 2012 Dec;188(6): $2152-7$ 
8 Barentsz JO, Richenberg J, Clements R, Choyke P, Verma S, Villeirs G, et al.; European Society of Urogenital Radiology. ESUR prostate MR guidelines 2012. Eur Radiol. 2012 Apr;22(4):746-57.

9 Soylu FN, Eggener S, Oto A. Local staging of prostate cancer with MRI. Diagn Interv Radiol. 2012 Jul-Aug;18(4):365-73.

10 Kim CK, Park BK, Han JJ, Kang TW, Lee HM. Diffusion-weighted imaging of the prostate at $3 \mathrm{~T}$ for differentiation of malignant and benign tissue in transition and peripheral zones: preliminary results. J Comput Assist Tomogr. 2007 May-Jun;31(3):449-54.

11 Tamada T, Sone T, Jo Y, Toshimitsu S, Yamashita T, Yamamoto A, et al. Apparent diffusion coefficient values in peripheral and transition zones of the prostate: comparison between normal and malignant prostatic tissues and correlation with histologic grade. J Magn Reson Imaging. 2008 Sep;28(3):720-6.

12 Pickles MD, Gibbs P, Sreenivas M, Turnbull LW. Diffusion-weighted imaging of normal and malignant prostate tissue at 3.0T. J Magn Reson Imaging. 2006 Feb;23(2):130-4.

13 McMahon CJ, Bloch BN, Lenkinski RE, Rofsky NM. Dynamic contrast-enhanced MR imaging in the evaluation of patients with prostate cancer. Magn Reson Imaging Clin N Am. 2009 May;17(2):363-83.

14 Demirtaş A, Sönmez G, Tombul ŞT, Demirtaş T, Akgün H. Comparison of the Upgrading Rates of International Society of Urological Pathology Grades and Tumor Laterality in Patients Undergoing Standard 12-Core Prostate Biopsy versus Fusion Prostate Biopsy for Prostate Cancer. Urol Int. 2019;103(3):25661.

15 Ghosh A, Heston WD. Tumor target prostate specific membrane antigen (PSMA) and its regulation in prostate cancer. J Cell Biochem. 2004 Feb;91(3):528-39.

16 Silver DA, Pellicer I, Fair WR, Heston WD, Cordon-Cardo C. Prostate-specific membrane antigen expression in normal and malignant human tissues. Clin Cancer Res. 1997 Jan;3(1):81-5.

17 Ceci F, Castellucci P, Cerci JJ, Fanti S. New aspects of molecular imaging in prostate cancer. Methods. 2017 Nov; 130:36-41.
18 McCarthy M, Langton T, Kumar D, Campbell A. Comparison of PSMA-HBED and PSMAI\&T as diagnostic agents in prostate carcinoma. Eur J Nucl Med Mol Imaging. 2017 Aug; 44(9):1455-62.

19 Weineisen M, Schottelius M, Simecek J, Baum RP, Yildiz A, Beykan S, et al. 68Ga- and 177Lu-labeled PSMA I\&T: optimization of a PSMA-targeted theranostic concept and first proof-of-concept human studies. J Nucl Med. 2015 Aug;56(8):1169-76.

20 Weineisen M, Simecek J, Schottelius M, Schwaiger M, Wester HJ. Synthesis and preclinical evaluation of DOTAGA-conjugated PSMA ligands for functional imaging and endoradiotherapy of prostate cancer. EJNMMI Res. 2014 Dec;4(1):63.

21 American College of Radiology. Prostate imaging reporting and data systems (PI-RADS). Reston: ACR; 2019. Available from: https:// www.acr.org/Clinical-Resources/Reportingand-Data-Systems/PI-RADS.

22 D'Amico AV, Whittington R, Malkowicz SB, Schultz D, Blank K, Broderick GA, et al. Biochemical outcome after radical prostatectomy, external beam radiation therapy, or interstitial radiation therapy for clinically localized prostate cancer. JAMA. 1998 Sep;280(11): 969-74.

23 Amin MB, Edge FL, Edge SB, Stephen B, Edge $\mathrm{MD}$, et al. The eighth edition AJCC Cancer Staging Manual: continuing to build a bridge from a population-based to a more "personalized" approach to cancer staging. CA Cancer J Clin. 2017;67(2):93-9.

24 Gözen AS, Akin Y, Ates M, Hruza M, Rassweiler J. Impact of laparoscopic radical prostatectomy on clinical $\mathrm{T} 3$ prostate cancer: experience of a single centre with long-term follow-up. BJU Int. 2015 Jul;116(1):102-8.

25 Epstein JI, Egevad L, Amin MB, Delahunt B, Srigley JR, Humphrey PA; Grading Committee. The 2014 International Society of Urological Pathology (ISUP) Consensus Conference on Gleason grading of prostatic carcinoma: definition of grading patterns and proposal for a new grading system. Am J Surg Pathol. 2016 Feb;40(2):244-52.

26 Eiber M, Weirich G, Holzapfel K, Souvatzoglou M, Haller B, Rauscher I, et al. Simultaneous 68Ga-PSMA HBED-CC PET/MRI improves the localization of primary prostate cancer. Eur Urol. 2016 Nov;70(5):829-36.
27 Pinaquy JB, De Clermont-Galleran H, Pasticier G, Rigou G, Alberti N, Hindie E, et al. Comparative effectiveness of [(18) F]-fluorocholine PET-CT and pelvic MRI with diffusion-weighted imaging for staging in patients with high-risk prostate cancer. Prostate. 2015 Feb;75(3):323-31.

28 Tuğcu V, Akça O, Şimşek A, Yiğitbaşı İ, Yenice MG, Şahin S, et al. Robotic perineal radical prostatectomy and robotic pelvic lymph node dissection via a perineal approach: The Tugcu Bakirkoy Technique. Turk J Urol. 2018 Mar;44(2):114-8.

29 Berger I, Annabattula C, Lewis J, Shetty DV, Kam J, Maclean F, et al. 68Ga-PSMA PET/CT vs. mpMRI for locoregional prostate cancer staging: correlation with final histopathology. Prostate Cancer Prostatic Dis. 2018 Jun;21(2): 204-11.

30 Yilmaz B, Turkay R, Colakoglu Y, Baytekin HF, Ergul N, Sahin S, et al. Comparison of preoperative locoregional Ga-68 PSMA-11 PET-CT and mp-MRI results with postoperative histopathology of prostate cancer. Prostate. 2019 Jun;79(9):1007-17.

31 Kausik SJ, Blute ML, Sebo TJ, Leibovich BC, Bergstralh EJ, Slezak J, et al. Prognostic significance of positive surgical margins in patients with extraprostatic carcinoma after radical prostatectomy. Cancer. 2002 Sep; 95(6):1215-9.

32 Winter A, Kneib T, Wasylow C, Reinhardt L, Henke RP, Engels S, et al. Updated nomogram incorporating percentage of positive cores to predict probability of lymph node invasion in prostate cancer patients undergoing sentinel lymph node dissection. J Cancer. 2017 Aug;8(14):2692-8.

33 Petersen LJ, Nielsen JB, Langkilde NC, Petersen A, Afshar-Oromieh A, et al. (68) GaPSMA PET/CT compared with MRI/CT and diffusion-weighted MRI for primary lymph node staging prior to definitive radiotherapy in prostate cancer: a prospective diagnostic test accuracy study. World J Urol. 2020;38(4): 939-48.

34 Hope TA, Goodman JZ, Allen IE, Calais J, Fendler WP, Carroll PR. Metaanalysis of 68Ga-PSMA-11 PET accuracy for the detection of prostate cancer validated by histopathology. J Nucl Med. 2019 Jun;60(6):786-93. 